\title{
Oxygen Profile in an experimental Cell Culture Apparatus
}

\author{
C.J.W. Breward ${ }^{1} \dagger$, and D.F. PARKer ${ }^{2}$ \\ ${ }^{1}$ School of Mathematical Sciences, University of Nottingham \\ ${ }^{2}$ Mathematics and Statistics Department, University of Edinburgh
}

(Communicated to MIIR on 4 November 2021)

Study Group: ESGI 37, 10-14 April, 2000, Sheffield, UK

Communicated by: Chris Breward

Industrial Partner: Medisys PLC, Aberdeen.

Presenter: D.P. Donovan, Department of Pathology, University of Sheffield Medical School

Team Members: J. Bosson; H. Byrne, University of Oxford; J. DeWynne, University of Oxford; D. Donovan, University of Sheffield; A. Grief; B. Gillies; S.D. Howison, University of Oxford; J.R. King, University of Nottingham; J. Oliver; C.P. Please, University of Southampton W. Weinelt, Chemnitz University of Technology

Industrial Sector: Biomedical/Healthcare

Key Words: Hypoxia, Cell culture, Tumour cells, Oxygen transport

MSC2020 Codes: 92

\section{Summary}

Cells can be readily grown outside of the body on the bottom of specialised plastic tissue culture dishes containing a nutrient liquid. The use of culture dishes with an oxygen-permeable base allows oxygen to reach the cells from below. In particular experiments, an impermeable glass slip covered with tumour cells is placed in the centre of a permeable culture dish and endothelial cells are seeded on the base of the dish around the outside of the cover slip. Our aim is to determine whether the tumour cells at the centre become hypoxic.

We build and solve mathematical models for the transport of oxygen in this cell culture apparatus. Given the physical parameters, we determine that the original set is likely to be sub-optimal. We propose revised geometries of the apparatus that result in better concentration gradients.

$\dagger$ Corresponding Author: breward@maths.ox.ac.uk 


\title{
Oxygen profile in an experimental cell culture apparatus
}

\author{
C.J.W. Breward ${ }^{* \dagger}$ and D.F. Parker ${ }^{\ddagger}$
}

Problem presented by D.P. Donovan from Department of Pathology, University of Sheffield Medical School, Sheffield and Medisys PLC, Aberdeen.

\section{The brief}

"Various cell types can be readily grown outside of the body in specialised plastic tisue culture dishes. Cells are grown on the bottom of such dishes and a nutritious liquid medium added above them. Under normal culture conditions oxygen enters the culture dishes via small spaces under the lid and diffuses into the medium to provide the cells with oxygen. When the depth of the medium is low, a reasonable level of oxygen reaches the cells on the floor of the dish to enable them to grow. Use of culture dishes with an oxygen-permeable base allows oxygen to access the cells from below. We hypothesize that if part of the base is covered by a glass coverslip (not permeable to oxygen) coated with cells on its upper surface, these cells will experience an oxygen gradient, with the cells at the edge of the coverslip being well-oxygenated and those at the centre quickly becoming hypoxic (low in oxygen) due to limited diffusion inwards from the edge (i.e. exposed to oxygen entering through the base of the culture dish). This project will draw on known diffusion characteristics and cell consumption rates for oxygen to predict oxygen profiles in this particular apparatus and allow determination of the most suitably sized disc to create a marked hypoxic region at the centre. This will be invaluable in our subsequent experiments".

* Deparment of Pathology, University of Sheffield

${ }^{\dagger}$ School of Mathematical Sciences, University of Nottingham

$\ddagger_{\text {Mathematics and Statistics Department, University of Edinburgh }}$ 


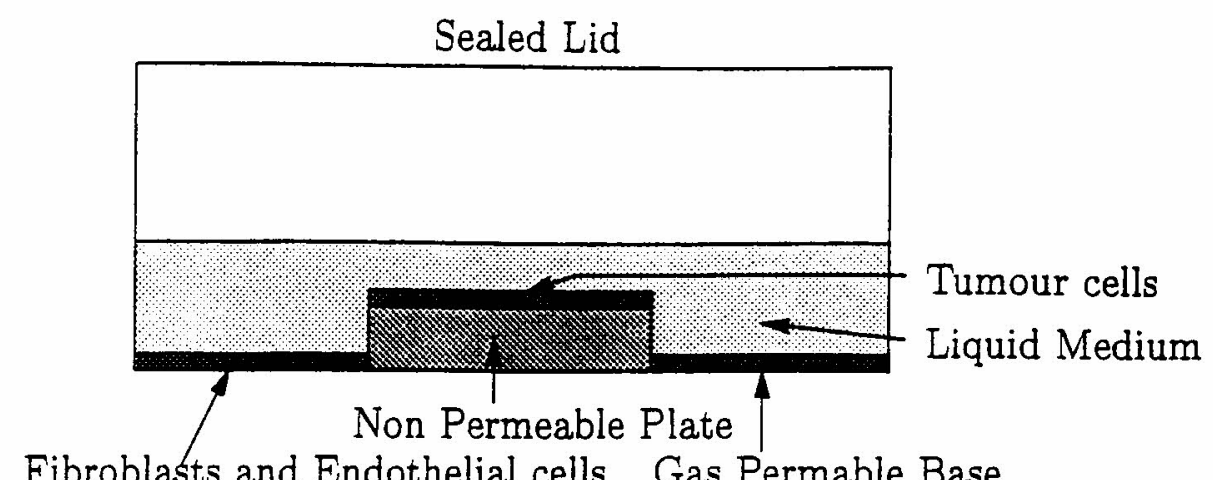

Figure 1: The experimental set up

\section{Interpretation and Aims}

The idea behind the experiment is to subject some of the tumour cells on the coverslip to low levels of oxygen (hypoxia), while supplying to the endothelial cells and fibroblasts sufficient oxygen for them to remain viable. Under these conditions, the tumour cells produce growth factors. These factors bind to the endothelial cells and stimulate their proliferation, differentiation (into tubules) and migration (towards the source of the growth factor).

Our aims are to determine (i) whether the oxygen level above the tumour cells becomes hypoxic, and (ii) what the concentration profile would be. Also of interest is the concentration profile of the growth factors produced by the hypoxic tumour cells.

\subsection{Other experimental considerations}

There are a number of further pieces of information that we detail below.

- The experiment is supposed to run for a week.

- The liquid medium is present in order to supply nutrients to the tumour cells and endothelial cells. It needs replenishing at intervals of between 1 and 3 days. The medium is expensive.

- The dish has radius $25 \mathrm{~mm}$ and height $10 \mathrm{~mm}$, the liquid medium is $2 \mathrm{~mm}$ deep, the glass plate raises the tumour cells $1 \mathrm{~mm}$ above the 
bottom of the dish, and the glass plate has radius of $7.5 \mathrm{~mm}$ (although other radii could be used).

- The gas in the "headspace" above the medium is air.

- The oxygen concentration below the permeable plate is atmospheric.

- The tumour cells totally cover the coverslip.

- Dead tumour cells do not remain fixed to the coverslip: they float off into the liquid medium.

\section{Formulation of the mathematical model}

We assume that the oxygen diffuses through the liquid (with diffusivity $D$ ) and through the gas in the headspace (with diffusivity $D_{1}$ ), is supplied through the permeable membrane with mass transfer coefficient $k$, and is consumed by the fibroblast-endothelial cell mix which we will henceforth call the "stromal mix" at a rate associated with mass transfer coefficient $\alpha$. Oxygen is also consumed by the tumour cells at a rate associated with mass transfer coefficient $\beta$. We show a schematic representing the model in Figure 2. We denote the concentration of oxygen in the liquid by $C$ and the concen-

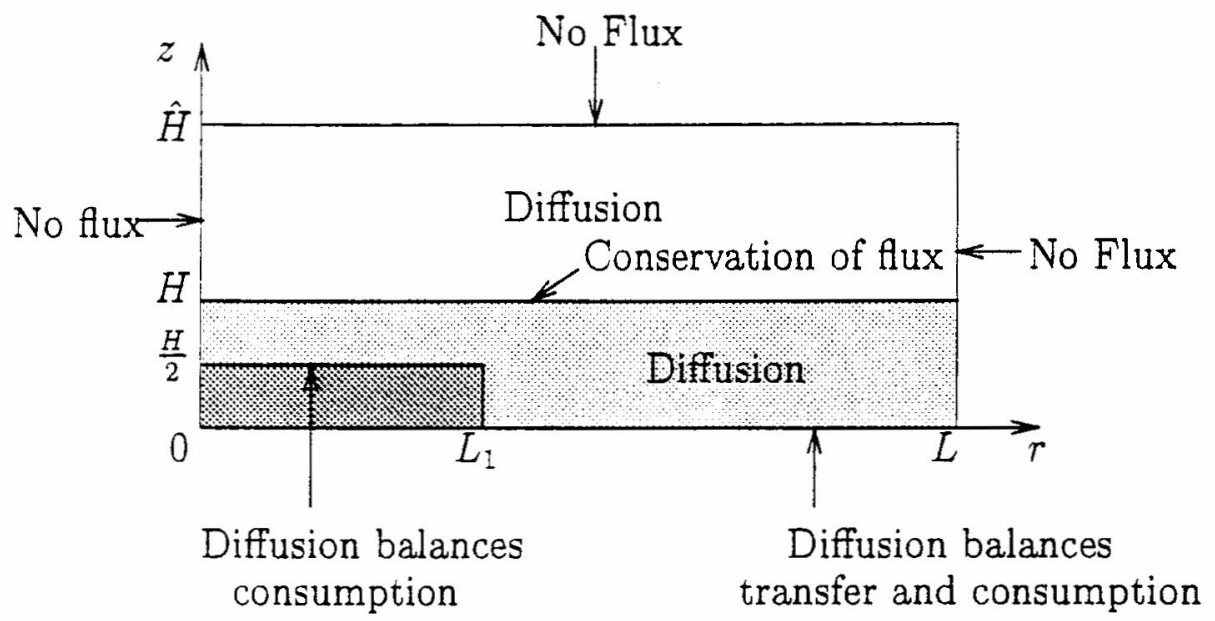

Figure 2: Schematic of the experimental set-up showing, in words, the mathematical model. 
tration of oxygen in the headspace by $\hat{C}$. Thus, in the absence of convection the model reads

$$
\begin{gathered}
C_{t}=D \nabla^{2} C, \quad \text { in the liquid, } \\
\hat{C}_{t}=D_{1} \nabla^{2} \hat{C}, \quad \text { in the headspace, }
\end{gathered}
$$

with

$$
\begin{array}{rlrl}
C_{r}=0 \text { at } r=L_{1}, L(0<z<H / 2) & \text { and } & r=0, L(H / 2<z<H), \\
+D C_{z}=k\left(C-C_{\text {out }}\right)+\alpha C & \text { on } & & z=0\left(L_{1}<r<L\right) \\
-D C_{z}=K^{*}\left(C-C_{\text {head }}\right) & \text { on } & z & =H(0<r<L) \\
+D C_{z}=\beta C & \text { on } & z & =H / 2\left(0<r<L_{1}\right), \\
C_{z}=0 & \text { on } & z & =\hat{H} \\
C=C_{\text {out }} & \text { at } & t & =0 .
\end{array}
$$

Before proceeding to discuss the sizes of all the parameters in the model, we comment that $C_{\text {out }}$ is not the same as $C_{\text {atm }}$ (the concentration of oxygen in the atmosphere). This is because at an air-liquid interface, the oxygen at both sides of the interface must have the same "chemical potential". This results in $C_{\text {out }}$ being significantly below $C_{\text {atm }}$. Appropriate values are $C_{a t m}=8 \mathrm{~mol}$ $\mathrm{m}^{-3}$, while $C_{\text {out }}=3 \mathrm{~mol} \mathrm{~m}^{-3}$. Similarly, $C_{\text {head }} \neq \hat{C} ; C_{\text {head }}$ is the oxygen concentration in the liquid which has the same chemical potential as the oxygen concentration $\hat{C}$ in the headspace.

We note that we have not formulated an equation for the evolution of the tumour cells. We assume that they continue to form a monolayer on top of the coverslip, and that, when a tumour cell dies, it floats away in the liquid medium, and proliferation occurs within the monolayer so as to fill the space vacated.

\subsection{Parameter values}

Here we list the relevant parameter values for the problem

$$
\begin{aligned}
\hat{H} & =10 \mathrm{~mm}, \\
H & =2 \mathrm{~mm},
\end{aligned}
$$




$$
\begin{aligned}
L & =25 \mathrm{~mm}, \\
L_{1} & =7.5 \mathrm{~mm}, \\
C_{\text {out }} & =3 \mathrm{~mol} \mathrm{~m}^{-3}, \\
C_{\text {atm }} & =8 \mathrm{~mol} \mathrm{~m}^{-3}, \\
C_{\text {hyp }} & =0.5 \mathrm{~mol} \mathrm{~m}^{-3} \\
D & =3.4 \times 10^{-9} \mathrm{~m}^{2} \mathrm{~s}^{-1}, \\
k & =5.8 \times 10^{-6} \mathrm{~m} \mathrm{~s}^{-1} \\
\alpha & =5 \times 10^{-9} \mathrm{~m} \mathrm{~s}^{-1}, \\
\beta & =2 \times 10^{-8} \mathrm{~m} \mathrm{~s}^{-1},
\end{aligned}
$$

where $C_{h y p}$ is the oxygen concentration below which the cells experience "hypoxia". We note that $k^{*}$ is unknown, but we assume it to be of magnitude comparable to $k$. Also, $D_{1}$ is not specified, but is assumed to be much bigger than $D$.

\subsection{Nondimensional model (in the liquid)}

We nondimensionalize using

$$
\begin{array}{ll}
C=C_{\text {out }} C^{\prime}, & r=L_{1} r^{\prime}, \\
z=H z^{\prime}, & t=T t^{\prime} .
\end{array}
$$

Then, after primes ' are dropped, the model in the liquid reads (in dimensionless variables)

$$
C_{t}=P_{1}\left(\frac{1}{r}\left(r C_{r}\right)_{r}+\frac{1}{\epsilon^{2}} C_{z z}\right)
$$

with

$$
\begin{array}{rll}
C_{r}=0 & \text { on } & r=0, r_{1}(1 / 2<z<1), \\
C_{z}=\tilde{\beta} C, & \text { on } & z=1 / 2,(0<r<1), \\
C_{z}=\tilde{k}(C-1)+\tilde{\alpha} C & \text { on } & z=0\left(1<r<r_{1}\right), \\
C_{z}=-\tilde{k}^{*}\left(C-C_{\text {head }}\right) & \text { on } & z=1\left(0<r<r_{1}\right), \\
C_{r}=0 & \text { on } & r=1, r_{1}(0<z<1 / 2), \\
C=1 & \text { at } & =0,
\end{array}
$$

where we have introduced 7 nondimensional parameter groups

$$
\epsilon=\frac{H}{L_{1}}, \quad \tilde{\beta}=\frac{\beta H}{D}, \quad P_{1}=\frac{D T}{L_{1}^{2}},
$$




$$
\tilde{\alpha}=\frac{\alpha H}{D}, \quad \tilde{k}, \tilde{k}^{*}=\frac{k H}{D}, \frac{k^{*} H}{D}, \quad r_{1}=\frac{L}{L_{1}} .
$$

We note that the timescale $T$ is not yet chosen. This is because there are two possible choices, which correspond to a vertical diffusion timescale $\left(T_{v}=\right.$ $\left.\epsilon^{2} L_{1}^{2} / D\right)$ and a horizontal diffusion timescale $\left(T_{h}=L_{1}^{2} / D\right)$. We shall discuss these below.

\section{The original model - why it won't work}

Using the parameter values given in $\S 3.1$, we calculate that $\epsilon \sim 0.08, \tilde{\beta} \sim$ $0.012, \tilde{k} \sim 1.7, \tilde{\alpha} \sim 2.9 \times 10^{-3}$ and $r_{1}=3.3$. Thus, since the aspect ratio $\epsilon$ is small, we may think of the region as long and thin. Since $\tilde{\alpha}$ and $\tilde{\beta}$ are small, consumption of oxygen by both the tumour cells and the stromal mix has negligible effect on the oxygen level. Thus, if we start in the configuration where $C=C_{\text {out }}$ in the liquid, $C=C_{\text {atm }}$ both in the headspace and below the permeable base, then the leading order solution to the nondimensionalized model is $C=1$ everywhere within the liquid, for all time, i.e. there is no change to the oxygen concentration with time, and no cells are subjected to hypoxia.

Suppose we now replace the air in the headspace with an inert gas and we remove all the oxygen from the medium before its addition to the dish. Then (16) is replaced by $C=0$ at $t=0$. The timescale for vertical diffusion through the liquid layer is $T_{v} \sim 20$ mins, using the parameters given in $\S 3.1$. Thus, on this timescale, there is an $O(1)$ change in the oxygen concentration. Since the diffusivity of oxygen in the atmosphere is much bigger than the diffusivity in the liquid, we assume that the oxygen concentration in the headspace is well mixed. Thus

$$
\hat{C}(t)=\frac{2 \pi D}{V} \int_{0}^{t}\left(\int_{0}^{L} C_{z} r \mathrm{~d} r\right) \mathrm{d} t,
$$

where $V$ is the volume of the headspace $\left(V=\pi L^{2}(\hat{H}-H)\right)$.

The steady state solution of the problem in this case also reads $C=1$, $\hat{C}=C_{a t m} / C_{\text {out }}$, and the steady state is approached on the timescale of vertical diffusion. Thus the preferred route for oxygen diffusion is shown in Figure 3 i.e., it diffuses vertically up through the liquid in $1<r<r_{1}$, mixes rapidly in the headspace and diffuses down towards the tumour cells. The timescale 


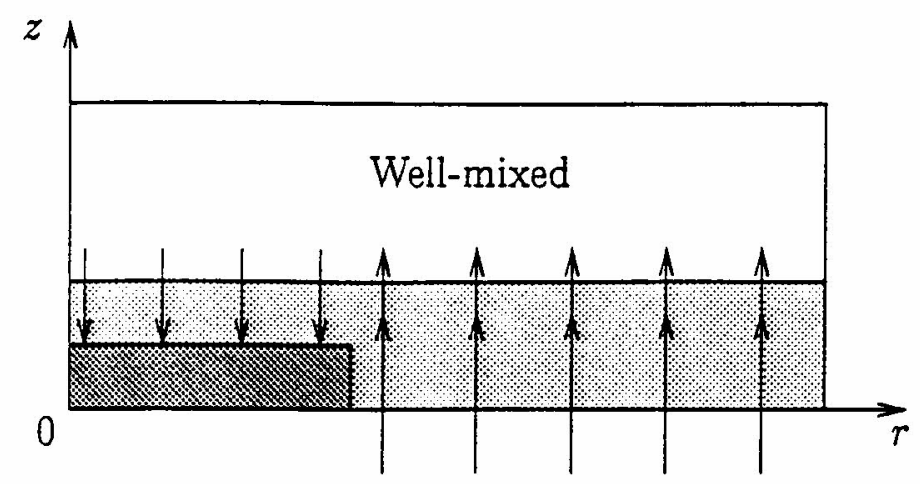

Figure 3: Schematic showing the oxygen path.

given by (17) for $\hat{C}(t)$ to reach $8 \mathrm{~mol} \mathrm{~m}^{-3}$ is approximately 5 hours (neglecting the diffusion back through the liquid to the tumour cells - which will not significantly increase the time). Thus we may conclude that, although the tumour cells are initially under hypoxia, the oxygen concentration rapidly rises to normoxic levels.

\section{Re-design of the apparatus}

We had several thoughts about redesigning the experimental arrangement.

- Leaving the top off and having an inert atmosphere above (so that the oxygen above the liquid is kept at zero concentration). This was rejected on experimental grounds, since leaving the top off the apparatus would encourage infection of the system by foreign bodies.

- Using much deeper liquid (so that the oxygen has to diffuse "a long way" through the liquid). This was rejected since the liquid medium is expensive.

- Preventing the diffusion of oxygen down from the headspace through the liquid to the tumour cells (so forcing oxygen to diffuse horizontally).

Thus we propose that, above the plate bearing the tumour cells, the lid is shaped to dip below the liquid surface. Two proposed designs of experimental apparatus are shown in Figure 4. Both involve modifying the lid. We must 

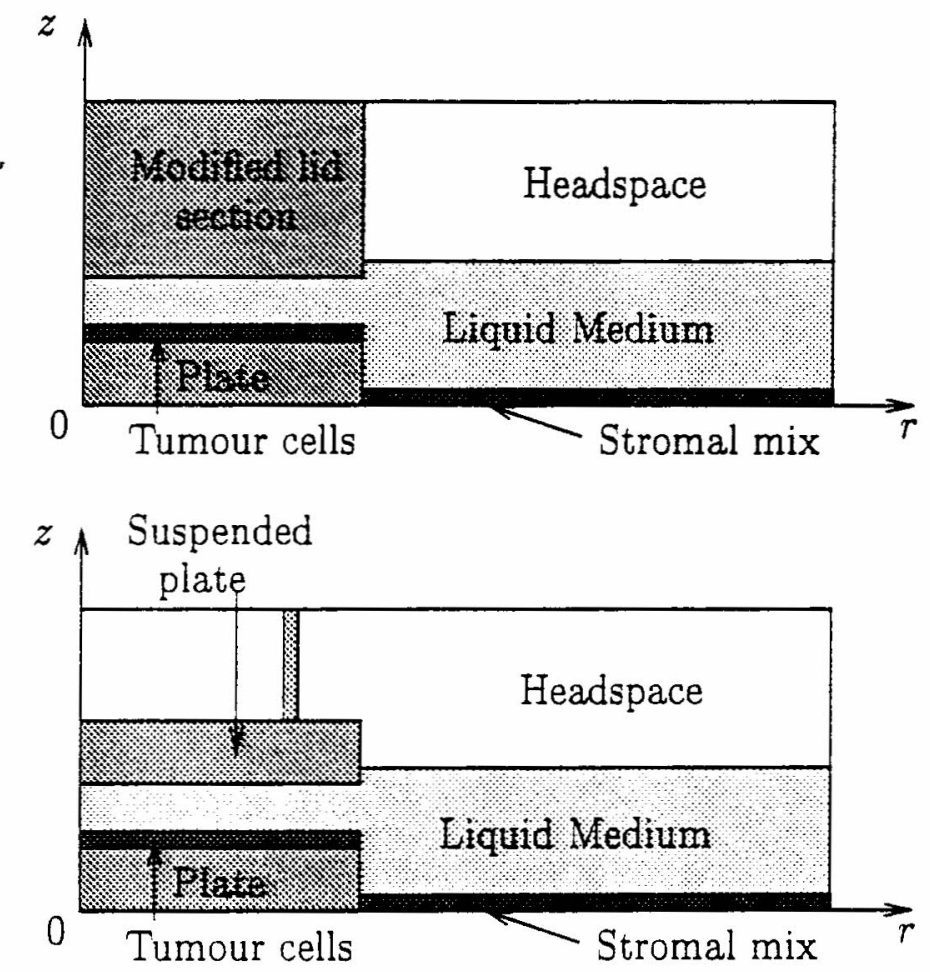

Figure 4: Possible re-designs of the experimental apparatus. 
then answer the question "What is the oxygen profile across the tumour cells in such a configuration?". To this end, we study the simplified problem: We suppose that the concentration in $1<r<r_{1}$ has equilibriated to $C=1$ and choose $T=L_{1}^{2} / D\left(=T_{h}\right)$, i.e., the horizontal diffusion timescale based on the radius of the thin disk above the tumour cells. Thus the problem in this region reads

$$
\begin{array}{rlrlrl}
C_{t} & =\frac{1}{r} & \left(r C_{r}\right)_{r} & +\frac{1}{\epsilon^{2}} C_{z z}, \\
C_{z} & =0 & \text { on } & & z & =1, \\
C_{z}=\tilde{\beta} C & \text { on } & & z & =1 / 2, \\
C_{r} & =0 & \text { at } & & r=0, \\
C & =1 & \text { at } & & r & =1 .
\end{array}
$$

We recall that $\tilde{\beta} \sim O\left(\epsilon^{2}\right)$, and we seek a solution in powers of $\epsilon^{2}$ and so set $C=C_{0}+\epsilon^{2} C_{1}+O\left(\epsilon^{4}\right)$. The $O(1)$ problem reads

$$
C_{0 z z}=0 \text { in } 1 / 2<z<1 \text { with } C_{0 z}=0 \text { on } z=1 \text {. }
$$

i.e. the solution is

$$
C_{0}=C_{0}(r, t) .
$$

Moreover, the condition at $z=1 / 2$ implies that $\tilde{\beta}=o(1)$. Thus, the equation governing $C_{0}(r, t)$ is found by writing $\bar{\beta}=\epsilon^{-2} \tilde{\beta}=\beta L_{1}^{2} / D H$ and then studying the $O\left(\epsilon^{2}\right)$ problem, which reads

$$
C_{0 t}=\frac{1}{r}\left(r C_{0 r}\right)_{r}+C_{1 z z}
$$

with

$$
\begin{array}{rll}
C_{1 z}=0 & \text { on } & z=1, \\
C_{1 z}=\bar{\beta} C_{0} & \text { on } & z=1 / 2 .
\end{array}
$$

Since this gives $C_{1 z}=-2 \vec{\beta}(z-1) C_{0}(r, t)$, the equation for $C_{0}$ thus reads

$$
C_{0 t}=\frac{1}{r}\left(r C_{0 r}\right)_{r}-2 \bar{\beta} C_{0},
$$

the last term acting as a distributed sink. The steady state solution to (28) with boundary conditions $(21),(22)$ is

$$
C_{0}=\frac{I_{0}(\sqrt{2 \bar{\beta}} r)}{I_{0}(\sqrt{2 \bar{\beta}})}
$$




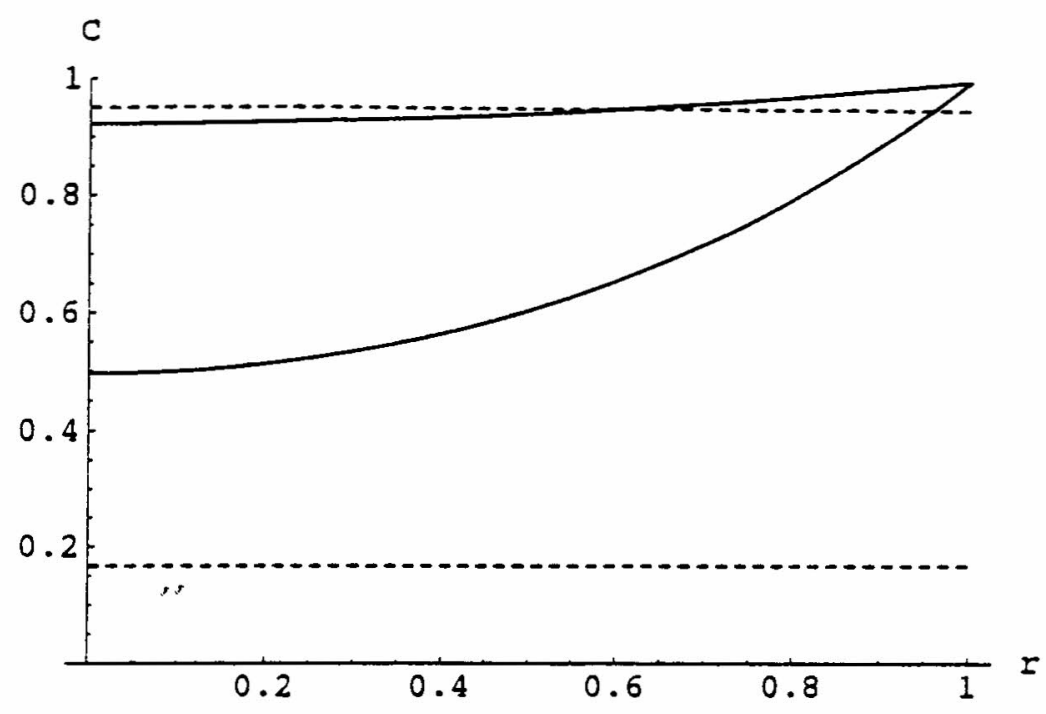

Figure 5: Graph showing $C_{0}$ for layer thickness $h=1 \mathrm{~mm}(H=2 \mathrm{~mm})$, $L_{1}=7.5 \mathrm{~mm}$ (top curve) and for a thinner layer $h=0.1 \mathrm{~mm}, L_{1}=7.5 \mathrm{~mm}$ (bottom curve). The dashed lines show the hypoxic level (i.e. the hypoxic oxygen tension $\left(=0.5 \mathrm{~mol} \mathrm{~m}^{-3}\right) /$ the external oxygen tension).

where $I_{0}$ is a modified Bessel's function. In Figure 5, this solution (29) for $C_{0}$ is plotted, together with a dashed line showing the level of oxygen required for the system to be hypoxic. We note that it is possible to control the atmosphere outside the apparatus, so that we are at liberty to vary $C_{\text {out }}$ if we need to (NB: decreasing $C_{\text {out }}$ moves the hypoxia line upwards in Figure 5). From the graph, we can see that, with the original choices for the plate size and liquid layer thickness, there is not much drop in concentration between the edge and the centre of the tumour mass. If we wish to use these parameters, we would thus have to decrease the external oxygen concentration to only fractionally above the hypoxic level. Decreasing the thickness of the liquid layer reduces the central oxygen tension, but such changes are experimentally unmanagable, since a small thickness of liquid will not provide sufficient nutrients for the tumour cells to survive.

\section{Conclusions}

Covering the tumour cells results in a gradient in the oxygen concentration across the tumour cells. The drop in overall magnitude, however, is small. 


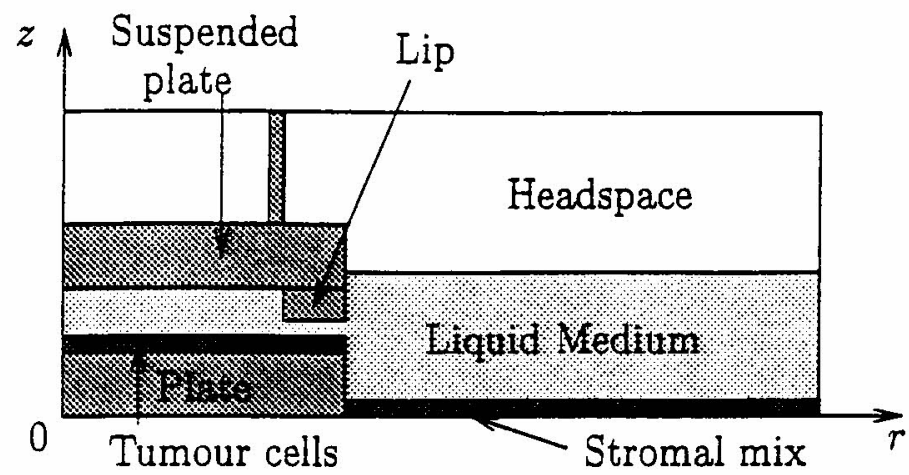

Figure 6: Possible re-designs of the experiment - including a lip.

Thus if some of the tumour cells are to experience hypoxia, the value of the concentration at the edge of the tumour cells must be only just above the hypoxic cut-off.

We have shown the oxygen profile in the steady state. Obviously, starting the system from zero oxygen concentration would be preferable to starting with the steady state level, since then the steady state will be approached from below (and the cells will experience more hypoxic stress).

\subsection{Use of a non-planar lid}

We can further reduce the oxygen concentration at the centre of the tumour cells by "choking" the oxygen out of the system. To do this, we propose introducing a small annular "lip" above the outer rim of the tumour layer, as shown in Figure 6. By introducing the lip, we are able to retain a reasonable volume of medium above the tumour cells, so that they can survive, while creating a sharper dependence of $C_{0}$ upon $r$ near $r=1$. In order to estimate whether such a change in the geometry of the lid makes a significant difference to the central oxygen tension, a simplified analysis is to merely patch together two solutions of the previous problem with different values of $\bar{\beta}$ (and with continuity of $C_{0}$ and of flux). We show such solutions in Figure 7 . Thus we can see that introducing the lip reduces the central value of the oxygen concentration - but not by a particularly significant amount (using a rim height of $0.1 \mathrm{~mm}$, which is the smallest that can be experimentally obtained). For lids of more general height variation, (28) is replaced by an equation with coefficients having different $r$-dependence, but we would expect transitions 


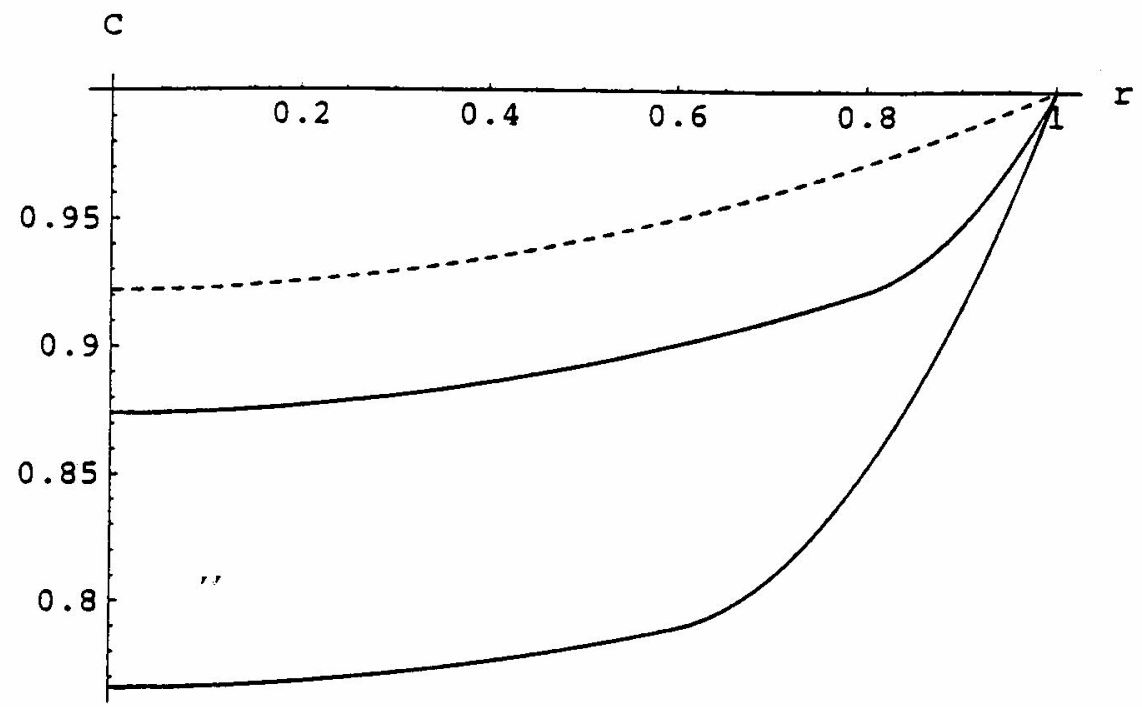

Figure 7: Graphs comparing $C_{0}$ with the unchoked case (shown dashed). The upper line has a $h=0.1 \mathrm{~mm}$ region extending across the outer $20 \%$ of the plate radius, while the lower dashed line has a $h=0.1 \mathrm{~mm}$ region extending across $40 \%$ of the plate.

to be less pronounced than in Figure 7.

\subsection{Reversing the position of the tumour cells and en- dothelial cells/fibroblasts.}

The radially symmetric geometry has the effect of accentuating the oxygen flux in the central region (as compared to the flux that would be obtained in a two-dimensional geometry). We may take advantage of this effect if we reverse the configuration by supplying oxygen at the centre of the dish, (i.e. by putting the fibroblast and endothelial cell mix in the centre of the dish and putting the tumour cells in a ring around the outside). The oxygen concentration is then reduced at larger radii by the diverging geometry, resulting in lower levels above the tumour cells. There are also other advantages in using this geometry: it uses more tumour cells (cheap) and fewer endothelial cells (expensive). Since there are also more tumour cells the oxygen concentration will be depleted even further than just by the geometric considerations. Any growth factors that are produced will also be focused as they diffuse inwards 


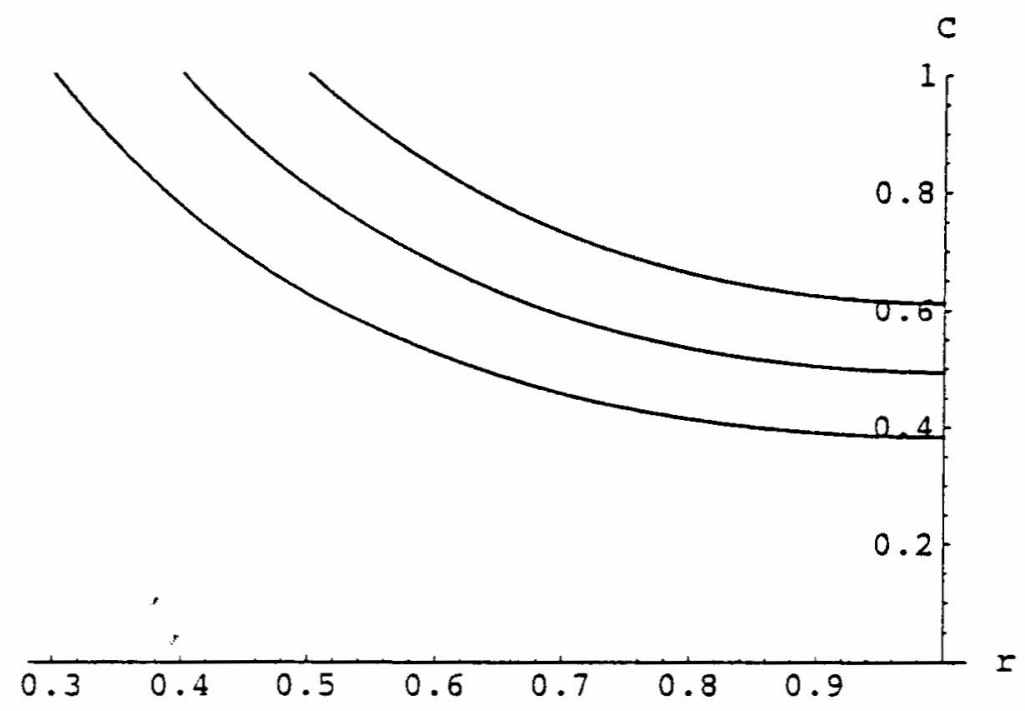

Figure 8: Graphs showing $C_{0}$ in the reversed geometry case. In all these cases, $L=25 \mathrm{~mm}$, and $H / 2=1 \mathrm{~mm}$. We vary the inner radius of the tumourbearing ring, where $C=1$. The upper curve has $r_{1}^{-1}=0.5\left(L_{1}=12.5 \mathrm{~mm}\right)$, the middle curve, $r_{1}^{-1}=0.4\left(L_{1}=10 \mathrm{~mm}\right)$ and the bottom curve, $r_{1}^{-1}=0.3$ $\left(L_{1}=7.5 \mathrm{~mm}\right)$.

from the tumour region. We now look at the steady state solution in this geometry, where the liquid above the annular plate bearing the tumour cells has depth $H / 2$ and occupies $1<r<r_{1}$.

The solution reads

$$
C=\frac{K_{1}\left(\sqrt{2 \bar{\beta}} r_{1}\right) I_{0}(\sqrt{2 \bar{\beta}} r)+I_{1}\left(\sqrt{2 \bar{\beta}} r_{1}\right) K_{0}(\sqrt{2 \bar{\beta}} r)}{K_{1}\left(\sqrt{2 \bar{\beta}} r_{1}\right) I_{0}(\sqrt{2 \bar{\beta}})+I_{1}\left(\sqrt{2 \bar{\beta}} r_{1}\right) K_{0}(\sqrt{2 \bar{\beta}})}, \quad 1<r<r_{1} .
$$

We show the solution in Figure 8.

We can easily see from the diagram that the oxygen tension in this case is much lower than with the cells in the original configuration, which means that the supply oxygen concentration can be greater than in the original case.

\subsection{Problems arising from the modified lids}

Unfortunately, introducing the depressed lid into these experiments also affects the diffusion of the growth factors away from the tumour cells through 
the liquid medium above the cell mix. The diffusivity of the growth factors is $D_{v}=1.4 \times 10^{-10} \mathrm{~m}^{2} \mathrm{~s}^{-1}$, which is much smaller than that for oxygen. In consequence, the associated timescales for diffusion of the growth factors are much larger than those for $\mathrm{O}_{2}$ (by a factor of 25). Thus, we need to check that a gradient of growth factors is set up in a reasonably short time. Suppose that the concentration of the growth factors is given by $v$, then the model reads

$$
\begin{array}{cccc}
v_{t}=D_{v} \nabla^{2} v & & \left(1<r<r_{1}\right), \\
v_{z}=0 & \text { on } & z=1, \\
v_{\tau}=0 & \text { on } & r=r_{1}, \\
D_{v} v_{z}=-\gamma & \text { on } & z=1 / 2,
\end{array}
$$

where $\gamma=2 \times 10^{-9} \mathrm{Kg} \mathrm{m}^{-2} \mathrm{~s}^{-1}$.

An appropriate nondimensional problem for $v^{\prime} \equiv D_{v}\left(\epsilon^{2} \gamma H\right)^{-1} v$ involves two time scales; vertical diffusion takes place over the timescale of $\tau=$ $D_{v} H^{-2} t$, while horizontal diffusion is associated with times $t^{\prime}=D_{v} L_{1}^{-2} t$ $\left(=\epsilon^{2} \tau\right)$. The corresponding nondimensional model in $1<r<r_{1}$ reads (after primes are dropped)

$$
\begin{array}{cccc}
v_{\tau}=v_{z z}+\frac{\epsilon^{2}}{r}\left(r v_{\tau}\right)_{r}, & \\
v_{z}=0 & \text { on } & z=1, \\
v_{\tau}=0 & \text { on } & r=r_{1}, \\
v_{z}=-\epsilon^{2} & \text { on } & z=1 / 2,
\end{array}
$$

together with a flux condition at $r=1$ matching to the deeper liquid.

Solutions may be sought in the form

$$
v=V(r, t)+\epsilon^{2} U(z, \tau),
$$

so giving

$$
\begin{gathered}
V_{t}=\frac{1}{r}\left(r V_{r}\right)_{r}+g_{0} \quad\left(1<r<r_{1}, \quad t>0\right), \\
U_{\tau}=U_{z z}-g_{0} \quad(1 / 2<z<1, \quad t>0),
\end{gathered}
$$

for some constant $g_{0}$, with initial conditions $V(r, 0)=0, U(z, 0)=0$. 
Equation (41) with $U_{z}=-1$ at $z=1 / 2, U_{z}=0$ at $z=1$ has the steady state solution $U=(z-1)^{2}$ provided that $g_{0}=2$. Then, the full solution for $U(z, \tau)$ is

$$
U=(z-1)^{2}+\frac{1}{2} a_{0}+\sum_{n=1}^{\infty} a_{n} \mathrm{e}^{-4 n^{2} \pi^{2} \tau} \cos 2 n \pi z
$$

where

$$
a_{n}=-4 \int_{1 / 2}^{1}(z-1)^{2} \cos 2 n \pi z d z
$$

so that $a_{n}=-(n \pi)^{-2}(n \geq 1)$ and $a_{0}=-1 / 6$. Thus

$$
U=(z-1)^{2}-\frac{1}{12}-\pi^{-2} \sum_{n=1}^{\infty} n^{-2} e^{-4 n^{2} \pi^{2} t / \epsilon^{2}} \cos 2 n \pi z .
$$

while $V(r, t)$ is the solution to

$$
V_{t}=\frac{1}{r}\left(r V_{r}\right)_{r}+2, \quad V_{r}\left(r_{1}, t\right)=0
$$

together with the initial condition $V(r, 0)=0$ and the flux condition at $r=1$.

Since the steady state solution to (45) is $\bar{V}=r_{1}^{2} \ln r-\frac{1}{2} r^{2}+V_{0} \equiv \bar{V}(r)$, the concentration of the growth factors tends on the timescale of $t$ towards the non-uniform state $\bar{V}(r)$, which has gradient $\bar{V}^{\prime}(r)=r_{1}^{2} r^{-1}-r$.

Endothelial cells detect gradients of growth factor when the background level is above a certain threshold $\sim 1 / 10$ of normal values. A lower bound on the time required to establish the required concentration gradient is provided by solving (45) together with the condition $V(1, t)=0$. In (45), set $V=$ $\bar{V}(r)+W(r, t)$, so that $r^{-1}\left(r W_{r}\right)_{r}-W_{t}=0$ with $W(r, 0)=-\bar{V}(r), W(1, t)=$ 0 and $W_{r}\left(r_{1}, t\right)=0$. Thus, $W$ is a linear combination of functions $\mathrm{e}^{-\mu_{n}^{2} t} W_{n}(r)$, where $W_{n}(r)$ is an eigenfunction satisfying

$$
\left(r W_{n}^{\prime}\right)^{\prime}+\mu_{n}^{2} r W_{n}=0, \quad W_{n}(1)=0, \quad W_{n}^{\prime}\left(r_{1}\right)=0 .
$$

It has the form (in terms of Bessel functions)

$$
W_{n}(r)=Y_{0}\left(\mu_{n}\right) J_{0}\left(\mu_{n} r\right)-J_{0}\left(\mu_{n}\right) Y_{0}\left(\mu_{n} r\right)
$$

where $\mu_{n}$ satisfies the eigenvalue equation $Y_{0}\left(\mu_{n}\right) J_{1}\left(\mu_{n} r_{1}\right)=J_{0}\left(\mu_{n}\right) Y_{1}\left(\mu_{n} r_{1}\right)$. Thus, the timescale for diffusion is $\mu_{0}^{-2}$, where $\mu_{0}$ is the (smallest) eigenvalue associated with the fundamental (monotonic) eigenfunction. 
Of course, we can get a crude estimate for the time for the central growth factor value to reach $1 / 10$ th of the value produced by the tumour cells collectively by solving the following problem on $0 \leq r \leq 1$ :

$$
v_{t}=\frac{1}{r}\left(r v_{\tau}\right)_{r},
$$

with

$$
\begin{array}{rll}
v=1 & \text { at } & r=1, \\
v_{r}=0 & \text { at } & r=0, \\
v=0 & \text { at } & t=0 .
\end{array}
$$

Here we are ignoring binding of the growth factor to the stromal mix, which is why we write down a problem independent of $z$. A simple numerical implementation of this problem reveals that $v(0, t) \sim 0.1$ by $t \sim 0.09$. Thus redimensionalising using the horizontal diffusion timescale, we find that the central concentration reaches $1 / 10$ th of the concentration at the rim of the tumour cells after about 10 hours (we are ignoring the time it takes to build up in the liquid above the tumour cells). Th

\section{Conclusions and recommendations}

We present the following conclusions and recommendations

- The original experimental set-up will not work: the oxygen concentration throughout the apparatus will equilibriate to the external level in a short time (compared to the time for biological processes).

- A lid should be used to prevent vertical diffusion from the headspace downward to the tumour cells.

- The change in oxygen tension between the centre of the tumour cells and the outer edge of the tumour cells is small even when the lid is present.

- In order that the tension falls into hypoxia above the tumour cells, the concentration of oxygen at supply should be reduced to only just above the hypoxic shut-off. 
- The radial symmetry of the system causes a raising of the oxygen tension (when compared to the two-dimensional case).

- A larger gradient in oxygen tension for a given supply tension and fluid layer thickness can be generated by reversing the positions of the two families of cells, i.e. putting the endothelial cell mix in the centre of the dish and the tumour cells round the outside.

- Capping the liquid with a lid also retards the diffusion of the growth factors.

- A detectable level of growth factors reaches the centre of this revised apparatus early in the experiment.

Report submitted by CJW Breward and DF Parker with contributions from A Grief.

Workshop contributions from J Bosson, H Byrne, J DeWynne, D Donovan, B Gillies, J Oliver, W Weinelt (and SD Howison, JR King, CP Please) 